\title{
BARRERAS Y DIFICULTADES ENFRENTADAS POR NIÑOS DE BÁSICA PRIMARIA FRENTE A LA EDUCACIÓN FÍSICA Y LOS DEPORTES
}

\author{
BARRIERS AND DIFFICULTIES OF PRIMARY EDUCATION \\ CHILDREN FACING PHYSICAL EDUCATION AND SPORTS
}

\author{
BARREIRAS E DIFICULDADES DAS CRIANÇAS DO ENSINO \\ FUNDAMENTAL EM RELAÇÃO À EDUCAÇÃO FÍSICA E ESPORTES
}

Ivonne Lorena Martínez G. ${ }^{1}$

\section{Resumen}

\begin{abstract}
Este artículo tiene como objetivo identificar las barreras y dificultades que enfrentan los niños de básica primaria frente a lo físico-deportivo y muestra el resultado parcial de una investigación mixta, en el campo de la educación física, adelantada como proyecto de grado titulado "Estrategias Tic para resolución de problemas físico-deportivos en niños(as) de 4.ำ del Liceo Campestre El Bosque”, en Mosquera, Cundinamarca. Los ejes temáticos fueron cuatro: 1) barreras y dificultades de aprendizaje fisico-deportivos; 2) pedagogía e intervención didáctica en educación física para resolver problemas en el aula; 3) Tecnologías de la información y la comunicación (TIC) como herramientas de aprendizaje; y 4) estrategias metodológicas de aprendizaje (didáctico participativo). La población objetivo estuvo conformada por 19 niñas y 9 niños de 9 a 10 años. Los instrumentos de indagación aplicados fueron una guía de entrevista y un diario de campo. Los aportes teóricos de Piaget, Ausubel, Vygotsky y Gardner apuntalaron la investigación en torno a que el aprendizaje fuese divertido y lúdico, para fomentar en los estudiantes la motivación. Los estudiantes y los padres de familia estuvieron comprometidos con las actividades para lograr los objetivos y la ejecución de acciones con resultados positivos, de acuerdo a los resultados se puede concluir que el docente debe aplicar procesos de enseñanza/ aprendizaje significativos y acordes con las necesidades del educando, teniendo en cuenta el contexto global. Las estrategias didácticas deben planearse y aplicarse con el apoyo de las TIC, y el proceso pedagógico debe ser balanceado y cercano a la educación física.
\end{abstract}

Palabras clave: aprendizaje; barreras; dificultades; fisico-deportivo; inclusión

Abstract

The purpose of this article is to identify the barriers and difficulties of primary education children in relation to physical activity and sports, and it shows the partial result of a mixed investigation, in the field of physical education, carried out as a degree project entitled "ICT Strategies for the resolution of physical-sports problems in children of the 4th year of the Liceo Campestre El Bosque", in Mosquera, Cundinamarca. The thematic axes were four: 1) physical and sports learning barriers and difficulties; 2) pedagogy and didactic intervention in physical education to solve problems in the classroom; 3) Information and communication technologies (ict) as learning tools; and 4) methodological learning strategies (participatory teaching). The target population was made up of 19 girls and 9 boys from 9 to 10 years old. The instruments of inquiry applied were an interview guide and a field diary. The theoretical contributions of Piaget, Ausubel, Vygotsky and Gardner underpinned the research around learning to be fun and playful, to encourage students with motivation. The students and parents were committed to the activities to achieve the objectives and the execution of actions with positive

1 Licenciada en Deporte y magíster en Educación. Universidad Internacional Iberoamericana. Universidad Pedagógica Nacional. Orcid: 0000-0003-3047-6280. magister.ivonne.martinez@gmail.com 
results. According to the results, it can be concluded that the teacher must apply significant teaching / learning processes in accordance with the needs of the educating, considering the global context. Teaching strategies must be planned and applied with the support of ICT, and the pedagogical process must be balanced and close to physical education.

Key words: learning; barriers; difficulties; physical activity; sports, inclusion

\section{Resumo}

O objetivo deste artigo é identificar as barreiras e dificuldades enfrentadas pelas crianças do ensino fundamental em relação ao aspecto físico-esportivo e mostra o resultado parcial de uma investigação mista, no campo da educação física, realizada como um projeto de graduação intitulado "Estratégias de Tic para a resolução de problemas físico-esportivos em crianças do $4^{\circ}$ ano do Liceo Campestre El Bosque, em Mosquera, Cundinamarca. Os eixos temáticos foram quatro: 1) barreiras e dificuldades de aprendizagem física e esportiva; 2) pedagogia e intervenção didática em educação física para resolver problemas em sala de aula; 3) Tecnologias da informação e comunicação (TIC) como ferramentas de aprendizagem; e 4) estratégias metodológicas de aprendizagem (ensino participativo). A população-alvo era composta por 19 meninas e 9 meninos de 9 a 10 anos. Os instrumentos de investigação aplicados foram um guia de entrevistas e um diário de campo. As contribuições teóricas de Piaget, Ausubel, Vygotsky e Gardner sustentaram a pesquisa em torno de aprender de uma maneira divertida e lúdica, para incentivar nos alunos a motivação. Os alunos e os pais se comprometeram com as atividades para atingir os objetivos e a execução de ações com resultados positivos e, de acordo com os resultados, pode-se concluir que o professor deve aplicar processos significativos de ensino / aprendizagem, de acordo com as necessidades dos alunos, levando em consideração o contexto global. As estratégias de ensino devem ser planejadas e aplicadas com o apoio das TIC, e o processo pedagógico deve ser equilibrado e próximo à educação física.

Palavras-chave: aprendizagem; barreiras; dificuldades; esporte-físico; inclusão

Fecha de recepción: 11 de enero de 2019

Fecha de evaluación: 01 de febrero de 2019

Para citar este artículo:

Martínez, L.I. (2019). Barreras y dificultades enfrentadas por niños de básica primaria frente a la educación física y los deportes. Lúdica Pedagógica, 29, 91-103. https://doi.org/10.17227/ludica.num29-11080 
En la actualidad, el aprendizaje desempeña un papel fundamental en el desarrollo de los educandos. Es un proceso que transita por experiencias positivas y negativas, por lo cual el docente debe estar atento a los intereses y necesidades de los educandos que tiene a su cargo. Los educadores cuentan con diversas teorías sobre el comportamiento humano, además de teorías del aprendizaje para la "adquisición de habilidades intelectuales, adquisición de información o conceptos, las estrategias cognoscitivas, destrezas motoras o actitudes" (Sarmiento, 2007, p. 3).

El proyecto que aquí se extracta surgió de la búsqueda de sustentos teóricos en los aportes y planteamientos de constructivistas como Piaget, Ausubel, Vygotsky y Gardner, entre otros, para quienes "el sujeto adquiere el conocimiento mediante un proceso de construcción individual, subjetivo y que sus expectativas y desarrollo cognitivo determinan en el niño la percepción del mundo" (citados por Sarmiento, 2007, p. 3).

El proyecto destacó la teoría de Piaget, el aprendizaje significativo de Ausubel, la teoría del procesamiento de la información de Gagné, el enfoque sociocultural de Vygotski (1931) y "los cambios producidos en los procesos mentales humanos, como consecuencia de la aparición de transformaciones en la organización social y cultural de la sociedad" (Sarmiento, 2007, p. 3). Se deben utilizar las herramientas necesarias para llevar el proceso de enseñanza/aprendizaje (EA), de acuerdo con la directriz de los teóricos que han estudiado el área de la educación, e incluir en ese proceso el uso de las tecnologías de la información y la comunicación (TIC).

Aunque para este artículo solo se desarrolla el primer objetivo, se formularon tres:

1. Identificar las barreras y las dificultades que presentan los niños en $4 .^{\circ}$ de básica primaria frente a lo físico-deportivo.

2. Aplicar estrategias lúdicas para la resolución de problemas físico-deportivos.

3. Implementar una propuesta pedagógica con el apoyo de herramientas TIC, para mejorar dificultades de aprendizaje fisico-deportivo.

Se aplicaron estrategias afectivas desde el docente hacia el educando. El primero se convirtió en participe de las experiencias originadas en las aulas, y ello fomentó en los estudiantes concentración, motivación y pensamientos positivos conducentes a su desarrollo.

La investigadora considera que la práctica de actividades físico-deportivas entre niños de básica primaria se ve afectada por barreras que dificultan la aplicación de sus programas, así como la actividad deportiva intra, extraescolar y el juego. Considera, además, que las dificultades presentes en el ámbito físico-deportivo impiden que los estudiantes realicen actividades que podrían ejecutar con ayuda de rutinas de internet, televisión y aplicaciones, por lo que dejan de lado la práctica o presentan escaso interés al respecto. Por su parte, la falta de aplicación de estrategias en los procesos de EA podría generar dificultades para los niños en cualquier área, pero con más énfasis en las cátedras de Educación Física y Deportes.

Según Ceballos, Medina, Ochoa y Carranza (2010) "Las barreras para la práctica de actividades físico-deportivas en escolares dificultan la aplicación de programas de educación física, actividad deportiva extraescolar y juego lúdico, lo que conlleva a problemas de salud, violencia verbal, de género y grupos vulnerables" (p. 1).

Con el objetivo de conocer las dificultades enfrentadas por los estudiantes de básica primaria en el Colegio El Bosque de Mosquera, Cundinamarca, en el campo de la formación deportiva, la investigadora aplicó instrumentos diagnósticos. Con estos, detectó, en la población objeto de estudio, barreras producto de la desigualdad de género, estrés y violencia escolar, entre otros que se relatan más adelante. Si bien sus causas son inciertas, las directivas y los docentes no las reconocen como actos de violencia, aunque realmente lo sean.

Por tal razón, este artículo describe las barreras y dificultades enfrentadas por niños de básica primaria frente a la educación física y los deportes. Presenta los resultados encontrados y su análisis, así como las conclusiones y algunas recomendaciones.

\section{METODOLOGÍA}

La investigación se adelantó en el Liceo Campestre El Bosque, ubicado en Mosquera Cundinamarca, institución que cuenta con más de 25 aulas dotadas 
tecnológicamente. Todos los estudiantes rotan por aquellas, excepto los de grado $4 \stackrel{0}{ }$ de básica primaria, que tienen un aula particular. La muestra la conforman 28 alumnos (19 son niñas y 9 niños), cuyas edades oscilan entre los 9 y los 10 años. La investigación es de carácter cuantitativo y cualitativo. No involucró variables ni tratamiento experimental. Es naturalista porque resalta los aspectos subjetivos del comportamiento de los niños de $4 \stackrel{0}{\circ}$ grado, su mundo, sus interacciones cotidianas y los significados de sus experiencias (André, citado en García de Sweet, 2017). La experiencia de los niños se mide por su interacción con los compañeros. Durante dicho proceso, se iban construyendo las interpretaciones, los significados y la visión de su realidad.

Como investigación cualitativa, se asumió un proceso activo e interactivo que relacionó las preguntas y los objetivos planteados como factores orientadores de la investigación. El investigador y el objeto de estudio se influyeron recíprocamente. El proyecto investigativo se apoyó en informes provenientes de entrevistas, encuestas, cuestionarios y observaciones que la investigadora relacionó en el cuaderno de notas de campo, mediante el método investigación-acción-participación (IAP), con el apoyo de las TIC, análisis de libros, entrevistas y encuestas.

\section{Técnicas e instrumentos para la} recolección de la información

De acuerdo con Stake (1999), parte de las técnicas e instrumentos aplicados durante la fase diagnóstica son una guía de entrevista (con la temática "Barreras de aprendizaje de la educación físico-deportiva”), para brindar una propuesta que permitiera solucionar el problema encontrado. Como parte del trabajo de intervención con los padres de familia, se les envió un enlace ${ }^{2}$ a un portal para que pudieran acceder a las estrategias utilizadas para trabajar con sus hijos.

Se formularon preguntas con respuestas cerradas que complementaron la información obtenida en el diario de campo y en la guía de observación, donde se registraron los comportamientos de los educandos durante el acto pedagógico (niños tímidos, agresores, agredidos), junto con datos obtenidos por la

2 https://lorenasport.wixsite.com/estudiogrado4 docente. Las entrevistas permitieron intercambiar ideas, palabras, actitudes y gestos significativos para analizar la información.

\section{INSTRUMENTOS Y HERRAMIENTAS}

Además de lo expresado para las entrevistas, en concordancia con lo revelado por Stake (1999), los datos se recogieron sin tener en cuenta un momento determinado. El proceso fue continuo, a partir de las percepciones, impresiones y vivencias en el aula, aunque inicialmente no se dispusiera de instrumentos concretos. Se acopiaron y registraron, informal y permanentemente, los datos en el cuaderno de campo, una fuente valiosa de información.

La técnica observación participante permitió a la investigadora involucrarse en la cotidianidad de sus alumnos, e interactuar con ellos, recogiendo sistemática y libremente los datos, de acuerdo con los planteamientos de (Taylor y Bogdan, 1986). Otras herramientas empleadas fueron las nuevas tecnologías de la información (NTIC). Las TIC, como el conjunto de la información (NTIC). Se usaron tabletas, programas informáticos, el portal presentado a los padres y alumnos (https://lorenasport.wixsite. com/estudiogrado4), teléfono, televisión, cámaras de video, correo electrónico, aplicaciones de audio y fotografías (junto con los permisos correspondientes), herramientas TIC que permitieron registrar el estudio.

\section{DESARROLLO}

Posiciones teóricas sobre los factores determinantes de la práctica deportiva y la actividad física

Según Moreno, Martínez y Alonso (2006), la actividad física en la infancia se muestra en acciones como el juego, caracterizado por la alegría y la espontaneidad. En concordancia con el constructivismo, y siguiendo a Vuori (1987), esta investigación considera que la actividad física es un medio de aprendizaje y autoexpresión requerido para el desarrollo físico, mental y social de los niños. Investigaciones como la de Ceballos et al. (2005) revelan que el factor edad también es un determinante de la actividad física, pues puede considerarse un impedimento personal. Una 
razón más para crear estilos de vida activos desde la escuela, que perduren en su vida futura.

Según el estudio de Treanor (1998), el 84,5 \% de los estudiantes analizados les gusta la educación física. Por su parte, Ceballos et al, (2010); Cervelló y Santos-Rosa (2000); y Moreno (2006) consideran que a los hombres les gusta más la asignatura, mientras que el gusto por la misma es menor en las mujeres, en las cuales desciende con el incremento de su edad porque se vuelven más sedentarias. Los hombres disfrutan del ejercicio por el hecho de competir, y este es un refuerzo inmediato que mantiene su conducta.

Zimmerman, Campomar, Fulugonio y Castro (2013) revelan que la:

Situación de un mundo masculino y femenino muy definidos ha cambiado y que hoy que ambos géneros están capacitados para realizar cualquier tipo de actividad o deporte... La nueva cultura del trabajo requiere de un conjunto de competencias cognitivas, sociales y tecnológicas, y es a través de estos conocimientos que se sustentan y orientan los procesos educativos (p. 5).

\section{Barreras y dificultades de}

aprendizaje físico-deportivo

Varios estudiosos han escrito sobre las barreras, problemas y dificultades de aprendizaje fisico deportivo. Por ejemplo, Ceballos et al. (2010) afirman que existen barreras para aplicar programas de educación física, actividad deportiva extraescolar y juego lúdico, y que esto colleva problemas de salud, violencia verbal y de género y la creación de grupos vulnerables. Ceballos revela que las oportunidades y las dificultades para participar en programas de actividad física se reconocen en excusas como falta de tiempo, de confianza o de dinero; carencia de éxito, pérdida de interés, demasiada presión, aburrimiento, miedo a las lesiones, falta de un sitio adecuado para prácticas, carencia de apoyo para actividades alternativas.

Los hallazgos de esta investigación se muestran en la tabla 1. Confirman los resultados de la percepción de la investigadora y de los docentes entrevistados sobre la relación entre pares en los niños de

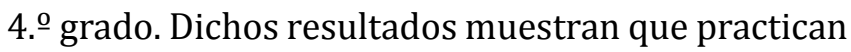
poca actividad deportiva o que no realizan actividad física como deberían: Los estudiantes afirman en sus entrevistas estar ocupados, no tienen tiempo, a su familia o amigos no les gusta hacer ejercicio, y que por eso no pueden hacer ejercicio en casa al igual en clase afirman estar cansados o que es riesgoso para su edad.

Las percepciones de las docentes sobre lo que piensan y expresan sus pupilos revelaron que los niños se contradicen cuando responden la pregunta: "Si contara con un lugar para hacer ejercicio, probablemente lo haría". Así, indican que la respuesta sería que sus "educandos nunca o pocas veces lo harían". Por su parte, destacan una concepción errada de sus estudiantes respecto al concepto descansar, cuando consideran que "es sinónimo de tardes de sofá". Más bien se trata de cambiar de actividad, introduciendo algún deporte que ayude al descanso físico y psicológico (ABC, 2013, p. 1).

Otras de las barreras para el aprendizaje fisico deportivo es el temor a los posibles riesgos de lesiones e inseguridades derivadas del contexto social. Según Devis (2000), los excesos de tiempo e intensidad en los ejercicios son los que generan lesiones, como sucede en el ciclismo, el esquí y el fútbol. Ceballos (2005) agrega que existen numerosas posibilidades, no solo para el disfrute, sino también para que los niños gocen de bienestar psicológico y social, aunque puedan sufrir golpes, raspones, moretones, fracturas y otras derivadas de la competitividad y el contexto social (Devis, 2000), o del exceso del tiempo y de la intensidad en el ejercicio (Serra, 2001). Los padres y los docentes se preocupan al respecto.

\section{Pedagogía e intervención didáctica en educación física}

Una forma común de entender la educación física en la sociedad actual es a través del juego y los deportes. Por ello, las percepciones de los docentes deben ser motivadoras y potenciadoras de actitudes y valores positivos. El enfoque metodológico de la educación física en primaria debe partir de una perspectiva lúdica, favorecida por la motivación innata de los niños hacia el juego y la actividad deportiva. Asimismo, debe enseñarles valores como la cooperación, el respeto por las reglas, el esfuerzo por superar las dificultades, la autonomía, la aceptación de roles y la alegría por sus logros (Lopez, 2012). Como dirían 
Piaget (1982) y Vigotsky (1931), los niños, mediante el juego, exploran su mundo físico, el ambiente social, perfeccionan los conceptos, amplían y "enriquecen el vocabulario, ejercitan su capacidad de atención y memoria, dan impulso a la imaginación y al pensamiento productor, mejoran su condición física y su capacidad para la aplicación funcional de destrezas y habilidades motrices en las más diversas situaciones" (Lopez, 2012, p. 1).

La teoría vigotskiana da forma y complementa el constructivismo moderno con las nuevas exigencias de un mundo globalizado que ya se introdujo en la educación. Acompañada de las teorías de Piaget, Ausubel y Bruner, entre otros, plantea un modelo en el que el docente impulsa en sus alumnos el desarrollo de competencias cognitivas, procedimentales $\mathrm{y}$ actitudinales.

Aber y Jones (2003) confirman que la educación física es básica: representa relaciones dinámicas favorecedoras para el desarrollo socioafectivo y posibilita la adquisición de actitudes positivas como la tolerancia y el respeto, que contribuyen a desarrollar la identidad del niño y un espíritu crítico. Lopez (2012) reconoce en la educación física "una multiplicidad de funciones que contribuyen por ellas mismas a la consecución de los objetivos generales de la educación obligatoria y que recogen todo el conjunto de prácticas corporales y no aspectos parciales de las mismas" (p. 2).

Hoyos Castro (2011) expresa que "la didáctica abarca el campo formativo en tanto se interese por la formación humana" (p. 1). La didáctica de la educación física, de acuerdo con Hernández Álvarez (2001), centra su estudio en las relaciones profesor-alumno, dentro de un proceso de enseñanza y aprendizaje intencional, en torno al movimiento humano como objeto de comunicación, con miras a que el alumno desarrolle sus actividades de aprendizaje en condiciones significativas y eficaces, por medio de acciones educativas concretas de intervención, orientada al proceso de la práctica más que al producto o excelencia atlética (Devis Devis, 2000). Agrega Hernández que el proceso de enseñanza y aprendizaje debe entenderse desde dos perspectivas: la teoría (que lleva a su explicación) y a la práctica (a su intervención). El objeto de la didáctica de la educación física se centra en el binomio "enseñanza y aprendizaje de las manifestaciones del movimiento humano", (p. 1) entendiéndolo no como un fenómeno físico, sino con intencionalidad y significado para el educando.

\section{La integración de las TIC a la educación física}

Está comprobado que las nuevas tecnologías son importantes para el deporte de alta competencia y para la educación. La integración de las Tic en la clase de educación física y en los deportes permite al alumno obtener las habilidades y las competencias básicas que todo alumno debe poseer y que todo profesional del deporte quiere promover en sus estudiantes. Así, la Ley General de Educación y la Ley 934 (Congreso de Colombia, Coldeportes, 2004) se crearon con "el fin de mejorar, innovar, potencializar y optimizar los procesos y quehaceres profesionales, ofreciendo así una visión práctica del desarrollo y la aplicación de los nuevos medios dentro del área de la actividad física y deportiva" (Mengual y Blasco, 2009, p. 1).

Las estrategias TIC son acciones pedagógicas que realiza el docente para producir aprendizajes significativos en sus educandos, a partir de sus necesidades e intereses, acordes con la sociedad actual, con el apoyo de la tecnología, la información y la comunicación (TIC). En palabras de Salinas (2004), para que esos procesos sean de calidad, requieren "cambios en la concepción de los alumnos-usuarios, cambios en los profesores y cambios administrativos en relación con el diseño y distribución de la enseñanza y con los sistemas de comunicación que la institución establece" (p. 1).

\section{RESULTADOS}

Los resultados de las entrevistas se observan en las tablas 1 y 2 .

Los resultados revelaron que los docentes en la institución no utilizaban las NTIC para resolver los problemas físico-deportivos de sus educandos. Estos datos justificaron, una vez más, la aplicación de la propuesta pedagógica que acompañó la investigación.

A la pregunta "¿Informa a los padres de familia las dificultades que presentaban sus estudiantes en educación-física y deportes?", todos respondieron que no. 
Tabla 1. Entrevista a profesores para descubrir problemas fisico-deportivos de sus estudiantes, indagar conductas y justificar por qué no realizan actividad física como deberían

\begin{tabular}{|l|c|c|c|c|}
\hline \multicolumn{1}{|c|}{ Conducta en el aula } & Nunca & Poco & Bastante & Demasiado \\
\hline $\begin{array}{l}\text { Siempre estoy ocupado, no tengo } \\
\text { tiempo para realizar actividad }\end{array}$ & & & 3 & \\
\hline $\begin{array}{l}\text { A mi familia y amigos no les gusta hacer } \\
\text { actividad física. No tengo oportunidad } \\
\text { de hacer ejercicio en casa. }\end{array}$ & & & 2 & 1 \\
\hline $\begin{array}{l}\text { Estoy cansado después del colegio } \\
\text { para hacer ejercicio. }\end{array}$ & & & 2 & 2 \\
\hline $\begin{array}{l}\text { No tengo acceso a caminos para trotar, } \\
\text { piscinas, senderos para bicicletas, etc. }\end{array}$ & 1 & & & \\
\hline Hacer ejercicio puede ser riesgoso para mi edad. & & & 1 & 2 \\
\hline Tengo poco tiempo libre para hacer ejercicio. & & & & 3 \\
\hline $\begin{array}{l}\text { No soy bueno en ninguna actividad } \\
\text { física para entretenerme }\end{array}$ & & & 1 & 2 \\
\hline $\begin{array}{l}\text { Si contara con un lugar para hacer } \\
\text { jejercicio, iprobablemente lo haría? }\end{array}$ & 1 & 12 & & \\
\hline
\end{tabular}

Fuente: elaboración propia con base en CDC.gov (2017); Ceballos et al. (2010), Gil (2015) y Stake (1999).

Tabla 2. Entrevista a docentes para conocer métodos de solución a problemas físico-deportivos enfrentados por sus estudiantes

\begin{tabular}{|c|c|c|c|c|}
\hline Profesores $4 .^{\circ}$ Liceo Campestre del Bosque & Si & No & \multicolumn{2}{|c|}{ ¿Cuáles? } \\
\hline $\begin{array}{l}\text { ¿Utiliza estrategias para resolución de problemas } \\
\text { físico-deportivos de los estudiantes? }\end{array}$ & 3 & & \multicolumn{2}{|c|}{$\begin{array}{l}\text { Aprendizaje guiado de básico } \\
\text { a complejo y motivación. }\end{array}$} \\
\hline $\begin{array}{l}\text { ¿Tiene en cuenta los intereres y necesidades de los } \\
\text { educandos al realizar lecturas y ejercicios deportivos? }\end{array}$ & 1 & 2 & & \\
\hline $\begin{array}{l}\text { ¿Utiliza las TIC como herramienta de trabajo para } \\
\text { enriquecer el gusto por los deportes de los estudiantes? }\end{array}$ & 0 & 3 & \multicolumn{2}{|c|}{ N.A } \\
\hline $\begin{array}{l}\text { ¿Informa a los padres de familia las dificultades que } \\
\text { presentan sus estudiantes en Educación Física y Deportes? }\end{array}$ & 0 & 3 & & \\
\hline \multirow{2}{*}{$\begin{array}{l}\text { ¿Qué dificultades o barreras ha visto en sus } \\
\text { alumnos para desarrollar su proceso de } \\
\text { aprendizaje de E. Física y Deportes? }\end{array}$} & $\begin{array}{c}\text { Falta } \\
\text { motivación }\end{array}$ & Cansancio & Otros & ¿Cuáles? \\
\hline & 3 & - & - & - \\
\hline
\end{tabular}

Fuente: elaboración propia con base en CDC.gov (s.f.), Ceballos et al. (2010) y Gil (2015).

Esta respuesta demuestra una falla de la relación escuela-familia. En coherencia con Alcalá Recuero, Martin Martínez y Ruiz Varela (2015), se considera que los docentes no tienen otro camino "para lograr el éxito educativo que contar con los padres para que exista coherencia entre lo que se hace en casa y en la escuela. Los padres no deberían educar a sus hijos al margen de los centros educativos" (p. 1). La tabla 3 establece el número de niños caracterizados como agresores, los mismos que contribuyen a generar en sus coeducandos problemas para el aprendizaje físico-deportivo. 
Tabla 3. Niños caracterizados como agresores (instrumento aplicado a estudiantes para descubrir problemas físico-deportivos)

\begin{tabular}{|l|c|c|c|c|}
\hline \multicolumn{1}{|c|}{ En el aula o colegio para molestar a un compañero: } & $\mathbf{1}$ & $\mathbf{2}$ & $\mathbf{3}$ & $\mathbf{4}$ \\
\hline Lo ignoras & 11 & 16 & 0 & 0 \\
\hline Lo insultas & 16 & 1 & 9 & 0 \\
\hline Le impides participar & 15 & 9 & 3 & 0 \\
\hline Le colocas apodos & 15 & 9 & 2 & 1 \\
\hline Hablas mal de él o ella & 15 & 9 & 1 & 1 \\
\hline Le escondes o dañas sus cosas & 24 & 3 & 0 & 0 \\
\hline Le robas sus cosas & 25 & 2 & 0 & 0 \\
\hline Le pegas & 25 & 2 & 0 & 0 \\
\hline Lo amenazas para que haga algo que no quiere hacer & 24 & 3 & 0 & 0 \\
\hline Te burlas de él & 24 & 3 & 0 & 0 \\
\hline
\end{tabular}

Fuente: elaboración propia con base en datos CDC.gov (2017), Ceballos et al. (2010) y Gil (2015).

Los resultados presentados en la tabla anterior permitieron percibir el modo como los niños se representan entre sí (relación entre pares). Asimismo, las entrevistas ayudaron a establecer datos relacionados con las categorías agredido y agresor. Se descubrieron problemas físico-deportivos y se comprobaron las percepciones de la investigadora para establecer el índice de niños agresores e indagar sobre la categoría exclusión social en el aula de clase o en la escuela. A la pregunta: "En el aula de clase o en el colegio, para molestar a un compañero, lo ¿ignoras?". Como respuesta, de 28 niños, 26 respondieron que a veces lo hacen; 11 indicaron que nunca lo hacen; 9 dijeron que, a menudo, insultan a algún compañero.

A la pregunta: "En el aula de clase o en el colegio, para molestar a un compañero, ¿le impides participar?: 3 niños respondieron que, a veces, les impiden participar; 3, a menudo; y 1, siempre. Para molestar a los niños agredidos, un grupo de 9 estudiantes (agresores) respondieron que les ponen apodos (a veces); 2 lo hacen a menudo; 2 roban las cosas a sus compañeros; dos les pegan; 3 los amenazan, y 3 se burlan de ellos.

Los resultados obtenidos durante las entrevistas revelan que, en el aula de la docente investigadora, hay al menos 2-3 alumnos agresores que someten a sus compañeros para molestarlos (acoso escolar). $\mathrm{Al}$ respecto, Gil Guzmán (2015) expresa que "el acoso escolar es un fenómeno que actualmente preocupa cada vez más a los centros educativos, se dice que cada vez hay más niños que suf ren algún tipo de abuso físico o psicológico dentro de la escuela por sus compañeros" (p. 2). A continuación, la tabla 4 presenta los resultados de la indagación por la categoría agresiones sufridas por los estudiantes, instrumento aplicado a estudiantes para descubrir problemas físico-deportivo.

Tabla 4. Agresiones sufridas por los estudiantes

\begin{tabular}{|l|c|c|c|c|}
\hline \multicolumn{1}{|c|}{$\begin{array}{c}\text { En el aula y/o colegio para molestarme } \\
\text { uno o varios compañeros }\end{array}$} & $\mathbf{1}$ & $\mathbf{2}$ & $\mathbf{3}$ \\
\hline Me ignoran & 11 & 13 & 2 & 1 \\
\hline Me insultan & 17 & 6 & 4 & 0 \\
\hline Me impiden participar & 12 & 14 & 1 & 0 \\
\hline Me colocan apodos & 17 & 9 & 0 & 1 \\
\hline
\end{tabular}




\begin{tabular}{|l|c|c|c|}
\hline \multicolumn{1}{|c|}{$\begin{array}{c}\text { En el aula y/o colegio para molestarme } \\
\text { uno o varios compañeros }\end{array}$} & $\mathbf{1}$ & $\mathbf{2}$ & $\mathbf{4}$ \\
\hline Hablan mal de mi & 10 & 15 & 2 \\
\hline Me esconden o dañan mis cosas & 16 & 9 & 2 \\
\hline Me roban las cosas & 19 & 7 & 1 \\
\hline Me pegan & 23 & 2 & 2 \\
\hline Me amenazan para que haga lo que no quiero hacer & 17 & 9 & 0 \\
\hline Me acosan sexualmente & 27 & - & 0 \\
\hline
\end{tabular}

Categoría: exclusión social

Nunca (1), A veces (2), a menudo (3) siempre (4).

Fuente: elaboración propia con base en datos de CDC.gov (s.f.), Ceballos et al. (2010) y Gil (2015).

Los datos mostraron hallazgos de agresores en la población estudiada. Concuerdan con los de la entrevista aplicada en la fase diagnóstica y con los de las tablas 3 y 4, que revelan hechos como el siguiente: "En el aula o en el colegio, para molestarme, uno o varios compañeros me ignora, me insulta, me esconde las cosas [...]". Más del $50 \%$ se declararon agredidos; solo 11 niños no se sienten ignorados, mientras que los 26 restantes sí; 13 respondieron "a veces"; 2 dijeron "a menudo"; y 1 respondió "siempre". Sobre los insultos, 10 los reciben (a veces, a menudo o siempre). A más de la mitad (55,5\%), les impiden participar, les colocan apodos, les esconden o roban las cosas. Estos resultados muestran la concurrencia de niños calificados en la categoría agredidos por los (agresores). Al respecto, Gil Guzman (2015, p. 2) afirma que "el abuso escolar e intimidación conocido como bullying ha sido investigado en muchos países, y actualmente existen numerosas documentaciones y programas de prevención e intervención" (p. 2).

Los resultados permitieron cumplir uno de los objetivos planteados en este trabajo: identificar las barreras y dificultades que presentan los niños en básica primaria en el ámbito físico-deportivo. A la vez, justificaron el segundo objetivo planteado, que buscaba aplicar estrategias lúdicas para resolver problemas físico-deportivos. Según lo que recomienda el Ministerio de Educación Nacional (2005), en su programa Colombia Aprende, los docentes, mediante el juego y la recreación, pueden lograr que los niños aprendan normas de convivencia básicas, a compartir con los demás, a aceptar al otro en su integridad, a valorar sus triunfos y a aceptar sus propias derrotas, y a trabajar en equipo para obtener metas comunes.

Con los hallazgos presentados en las tablas, se comprobó la necesidad de diseñar, presentar y aplicar como estrategia un proceso de intervención cognitivo conductual para los niños de 9-10 años, que cumple con el perfil de agresor escolar, como lo proponen Cerezo y Sánchez (2013). Estos autores recomidan un entrenamiento para controlar el comportamiento agresivo: se trata de hacerle ver al agresor que sus conductas de acoso no son permitidas, enseñándole prácticas socialmente apropiadas para expresar, de modo correcto, sus sentimientos. La dinámica incluye varias sesiones para trabajar sus pensamientos distorsionados y conductas difíciles de modificar (Cerezo y Sánchez, 2013).

En torno a la indagación sobre otras barreras, como la falta de práctica deportiva, la motivación y el escaso apoyo de los padres, aplicada a los estudiantes y sus padres, se encontró lo siguiente: de los 27 educandos, solo 15 expresan que habitualmente hacen ejercicio o algún deporte en casa, y 12 de ellos revelan que no hacen ningún tipo de ejercicio en casa. Solo a 14 niños les gustan los deportes, y solo 2 consideraron necesario practicar deportes; 6 niños practican deportes o ejercicios físicos porque los obligan, de lo que se deduce la falta de motivación para esa práctica. Los resultados obtenidos de la pregunta “¿Qué te gusta más: Educación Física o practicar deportes?" comprueban lo anterior: 12 niños respondieron que le gusta más la materia de Educación Física, y a 15 les gusta practicar un deporte. Por otro lado, ante la pregunta “ ¿Cuando observas ejercicios físicos 
sencillos, los comprendes?", más de la mitad respondió que sí, y diez respondieron que no. Esta última respuesta revela la necesidad de realizar, didácticamente, de manera clara y sencilla, las estrategias para una fácil comprensión de todos los niños.

Tabla 5. Gustos y hábitos deportivos

\begin{tabular}{|c|c|c|c|c|c|c|}
\hline \multirow{2}{*}{1} & \multirow{2}{*}{$\begin{array}{l}\text { Selecciona con } \mathrm{x} \text { el } \\
\text { área de conocimiento } \\
\text { que te gusta más }\end{array}$} & Matemáticas & Sociales & Español & Informática & Educ. Física \\
\hline & & 7 & 5 & 1 & 7 & 7 \\
\hline \multirow{2}{*}{2} & \multirow{2}{*}{$\begin{array}{l}\text { ¿Haces ejercicio o algún } \\
\text { deporte en casa? }\end{array}$} & si & no & & & \\
\hline & & 15 & 12 & & & \\
\hline \multirow[t]{2}{*}{3} & \multirow[t]{2}{*}{ Los deportes } & $\begin{array}{c}\text { ¿te gustan los } \\
\text { deportes? }\end{array}$ & $\begin{array}{l}\text { Necesitas } \\
\text { practicar } \\
\text { deportes }\end{array}$ & Te obligan & & \\
\hline & & 14 & 7 & 6 & & \\
\hline \multirow[t]{2}{*}{4} & \multirow[t]{2}{*}{ ¿Qué te gusta más? } & $\begin{array}{l}\text { Educación } \\
\text { física }\end{array}$ & $\begin{array}{c}\text { Practicar un } \\
\text { deporte }\end{array}$ & & & \\
\hline & & 12 & 15 & & & \\
\hline \multirow{2}{*}{5} & \multirow{2}{*}{$\begin{array}{l}\text { Si observas ejercicios } \\
\text { físicos sencillos ¿los } \\
\text { comprendes? }\end{array}$} & $\mathrm{Si}$ & No & & & \\
\hline & & 17 & 10 & & & \\
\hline \multirow{2}{*}{6} & \multirow{2}{*}{$\begin{array}{l}\text { ¿Cuánto tiempo te } \\
\text { ejercitas en casa? }\end{array}$} & $0 \mathrm{~min}$ & $15 \mathrm{~min}$ & $30 \mathrm{~min}$ & $60 \mathrm{~min}$ & \\
\hline & & 10 & 8 & 5 & 4 & \\
\hline
\end{tabular}

Fuente: elaboración propia.

En relación con el hábito deportivo de los padres, revelados en la tabla 5 , solo 10 (casi el $30 \%$ ) respondieron que sí tienen hábitos deportivos; 17 padres (más del $60 \%$ ) no tienen hábitos deportivos. A la pregunta " ¿Acostumbra a hacer ejercicio o deportes con su hijo?", un número elevado de padres (20) respondieron que no acostumbran a hacerlo. Esto se percibe como una razón o causa por la que no motivan a sus hijos. Solo 7 respondieron que sí tienen hábitos deportivos. A la pregunta “¿Comparte o participa en juegos con sus hijos?", la mayoría respondió que no, mientras que a la pregunta "¿A su hijo le infunde temor por sufrir una lesión al realizar un deporte?", 8 respondieron que sí.

Tabla 6. Indagación de hábitos deportivos de los padres de familia

\begin{tabular}{|c|c|c|c|c|c|}
\hline \multirow{4}{*}{$\begin{array}{l}\text { ¿Ud tiene hábitos } \\
\text { deportivos? }\end{array}$} & \multicolumn{2}{|c|}{$\mathbf{S i}$} & \multicolumn{2}{|c|}{ No } & \\
\hline & \multicolumn{2}{|c|}{10} & \multicolumn{2}{|c|}{17} & \\
\hline & Softball & Beisból & Fútbol & Otro & Ninguno \\
\hline & 2 & 5 & 10 & 3 & 7 \\
\hline \multirow{2}{*}{$\begin{array}{l}\text { ¿Cómo fomenta en sus } \\
\text { hijos el hábito deportivo? }\end{array}$} & \multicolumn{2}{|c|}{$\begin{array}{l}\text { Suministrar libros con } \\
\text { ejercicios para que los realice }\end{array}$} & \multicolumn{2}{|c|}{ Leyendo noticias deportivas } & \\
\hline & \multicolumn{2}{|c|}{8} & \multicolumn{2}{|c|}{19} & \\
\hline
\end{tabular}




\begin{tabular}{|l|c|c|}
\hline \multirow{2}{*}{$\begin{array}{l}\text { ¿Acostumbra hacer ejercicio } \\
\text { y/o deportes con su hijo? }\end{array}$} & 7 & Si \\
\cline { 2 - 3 } $\begin{array}{l}\text { ¿Comparte o participa } \\
\text { en juego con sus hijos? }\end{array}$ & 9 & 20 \\
\hline $\begin{array}{l}\text { ¿Infunde a su hijo } \\
\text { temor a una lesión por } \\
\text { realizar un deporte? }\end{array}$ & 8 & 18 \\
\hline
\end{tabular}

Fuente: elaboración propia con base en CDC.gov (s.f.), Ceballos et al. (2010) y Gil (2015).

$\mathrm{Al}$ analizar los fundamentos de intervención cognitivo-conductuales con el niño agresor en los casos de acoso, se encontró que los estudios concuerdan con lo revelado por Gil (2015). Los hallazgos para la población objeto de estudio y el análisis de los instrumentos aplicados en esta investigación permiten inferir que las conductas agresivas que conforman el acoso escolar entre los niños del Liceo Campestre El Bosque se corresponden con insultos, burlas, esconder o tomar los objetos de los coeducandos, y prácticas de exclusión como ignorarlos e impedirles jugar con los demás en el recreo. Tales conductas, expresa Gil (2015), por lo general son reforzadas por sus compañeros con risas y atención.

Durante la intervención, se encontró que los profesores ignoran el comportamiento inadecuado en clase, lo que permite que los niños agresores continúen con sus conductas agresivas, dado que las ganancias de tales conductas superan las sanciones y sus consecuencias.

Finalmente, en relación con la variable edad, la investigadora revela que, en torno al sexo, las diferencias en los ejercicios de niños y niñas en primaria son similares. Por tal razón, se pude estudiar conjuntamente para ambos sexos. Para Incheley et al. (2005); Ceballos, Medina, Ochoa, Carranza (2010); y Perula et al. (1998), la variable edad es un factor determinante de la actividad física, y puede considerarse un impedimento personal. Por el contrario, para Timón y Hormigo (2010) y Marcos (1989), las desigualdades se patentizan con la maduración sexual, momento en que las peculiaridades anatomofisiologías son evidentes y definitorias para ambos sexos.

\section{CONCLUSIONES}

$\mathrm{Al}$ indagar sobre las necesidades educativas de los niños en el campo físico-deportivo, los resultados revelaron barreras y dificultades enfrentadas por los estudiantes de $4^{\circ}$ grado. Los datos obtenidos se emplearon para diseñar una propuesta pedagógica para solucionar el problema

Según los cambios sociales y culturales actuales, el docente pude aplicar procesos de EA significativos, con intervenciones didácticas y pedagógicas que reduzcan las barreras frente a la educación física y lo deportivo.

Con herramientas TIC, se pueden reducir las barreras y los problemas físico-deportivos de los estudiantes. Las oportunidades educativas que ofrecen permiten mejorar la calidad de la educación que reciben, prevenir el fracaso, la exclusión social y otras posibles dificultades. Además, contribuyen a la participación de la familia en el sistema educativo de sus hijos, como lo expresan Alcalá, Martin y Ruiz (2015). En este estudio, los padres tuvieron la oportunidad de ingresar a un portal para participar en el proyecto llevado a cabo con sus hijos, mediante el enlace ttps://lorenasport.wixsite.com/ estudiogrado4. Se mostraron contentos y atentos con la información; manifestaron su acuerdo con las actividades y confirmaron haber visto los videos de sensibilización para implementar las cinco técnicas para un cambio de conducta, escritas por la docente con base en recomendaciones de De la Cruz Belerda (2016). 


\section{RECOMENDACIONES}

Se sugiere, para el desarrollo infantil, integrar las herramientas TIC y el juego en la educación físico-deportiva:

- Los programas de educación física, llevados a cabo con el uso de las CDC, generan en los niños un gusto inigualable por los aprendizajes y contribuyen a superar dificultades físico-deportivas.

- Promover los deportes y despertar en los niños un gran interés por ellos contribuye a fomentar su desarrollo integral.

\section{REFERENCIAS}

ABC.es (2013, 13 de febrero). Diez razones por las que los niños y adolescentes deben hacer deportes. http://www.abc.es/20120928/familia-vida-sana/ abci-diez-razones-correr-201209271642.html

Aber, L. y Jones, S. (2003). Trayectorias del desarrollo hacia violencia a medio chilhood: curso, diferencias demográficas y respuesta a intervención a base de escuela. Psicología del Desarrollo, 39(2), 324-348.

Alcalá Recuero, J., Martin Martínez, L. y Ruiz Varela, G. (2015, 2 de septiembre). La participación de las familias en el sistema educativo de sus hijos. Educaweb. http://www.educaweb.com/noticia/2015/02/09/participacion-familias-sistema-educativo-sus-hijos-8679/

CDC.gov (2017, 2 de septiembre). División de nutrición y actividad física de los CDC. https://www.cdc.gov/diabetes/ndep/pdfs/toolkits/camino-buena-salud/road-tohealth-barriers-quiz-spanish.pdf

CDC.gov (s.f.). Quiz de las barreras a la actividad física. ¿Qué cosas le impiden ser más activo? https://www.cdc.gov/ diabetes/ndep/pdfs/toolkits/camino-buena-salud/ road-to-health-barriers-quiz-spanish.pdf

Ceballos, G., Medina, R., Ochoa, F. y Carranza, G. (2010). Barreras para la práctica de actividades físico-deportivas en escolares. ArgenMex, 21. http://www.argenmex. fahce.unlp.edu.ar/5.-discapacidad-e-inclusion/19.-barreras-para-la-practica-de-actividades-fisico-deportivas-en-escolares

Ceballos, O. S. (2005). Gasto energético en escolares adolescentes de la ciudad de Monterrey. RESPIN, 6(3), 1-8.

Cerezo, F. y Sánchez, C. (2013). Eficacia del programa CIP para la mejora de la convivencia escolar y la prevención del bullying en alumnos de educación primaria. Madrid: Fuente Santana.

Cervelló, E. M. y Santos-Rosa, F. (2000). Motivación en las clases de educación física: un estudio de la perspectiva de las metas de logro en el contexto educativo. RPD PSicologia del Deporte, 9(12), 51-70.

Congreso de Colombia, Coldeportes $(2004,30$ de diciembre). Ley de Educacion Física: 934 de 2004. http://www.coldeportes.gov.co/normatividad/ normatividad_juridica/leyes/ley_934_2004

De la Cruz Belerda, N. (2017, 13 de septiembre). Modificación de conducta: 5 técnicas que reducen conductas rebeldes en niños. Cognifit: Health, Brain \& Neuroscience. https://blog.cognifit.com/es/ modificacion-de-conducta/

Devis Devis, J. (2000). Actividad fisica, deporte y salud. Zaragoza: INDE. https://www.iberlibro. com/9788495114099/Actividad-F\%C3\%ADsica-Deporte-Salud-Biblioteca-8495114097/ plp

García de Sweet, M. (10 de octubre de 2017). Métodos cualitativos de investigación. La investigación en educación. Academia. https://www.academia.edu/8569707/ UNIVERSIDAD_NACIONAL_EXPERIMENTAL_FRANCISCO_DE_MIRANDA_METODOS_CUALITATIVOS_EN_ LA_INVESTIGACION

Gil Guzmán, B. (2015). Intervención cognitivo-conductual con el niño agresor en un caso de acoso escolar. Psicología Clínica con Niños y Adolescentes, 2(1), 25-31. http:// www.revistapcna.com/sites/default/files/14-18_0.pdf

Hernández Álvarez, J. L. (2001). Didáctica de la educación física: reflexiones en torno a su estudio. Efdeportes.com, 7(42). https://www.efdeportes.com/efd42/ didacef.htm

Hoyos Castro, A. (enero de 2011). Algunos problemas didácticos en la clase de Educación Física. efdeportes. com, (152). http://www.efdeportes.com/efd152/problemas-didacticos-en-la-clase-de-educacion-fisica.htm

Incheley, J., Currie, D., Todd, J., Akhtar, P. y Currie, C. (2005). Persistent socio-demographic differences in physical activity among Scottish schoolchildren 1990-2002. European Journal of Public Health, 15(4), 386-388.

Lopez, F. (2012). Guía de la asignatura de grado «educación física en primaria ii» 2012/2013. Obtenido de Universidad de Murcia: https://aulavirtual.um.es/ umugdocente-tool/htmlprint/guia/RfRw9oqQknLa0mdBiQziJgucrhOQzxIrsVlX2bR9hGxYkfNmgkr 
Marcos Becerro, J. F. (1989). El niño y el deporte (el ejercicio en la promoción de la salud del niño y adolescente, desde la educación física a la alta competición). Revista de la Federación Española de Medicina, 784-802. DoI: 84-86541-02-6

Martínez, J., Rojas, C. y Duque, A. (2008). Agresividad en los escolares y su relación con las normas familiares. Revista Colombiana de Psiquiatría, 37(3), 365-377.

Mengual, S. y Blasco, M. J. (2009). TIC aplicadas a la educación física y el deporte. Universidad de Alicante. https:// ocw.ua.es/es/ingenieria-y-arquitectura/tic-aplicadasa-la-educacion-fisica-y-el-deporte-2009.html

Ministerio de Educacion Nacional (8 de abril de 2005). Colombia aprende.edu.co. http://www.colombiaaprende.edu.co/sites/default/files/naspublic/ guia_no._49_0.pdf

Moreno M., Martínez, G. y Alonso, V. (2006). Actitudes hacia la práctica físico-deportiva según el sexo del practicante. Revista internacional de Ciencias del Deporte (RICIDE), 2(3), 20-43.

Perula, L., Lluch, C., Ruiz, R., Espejo, J. y Tapia, G. (1998). Prevalencia de actividad física y su relación con variables sociodemográficas y ciertos estilos de vida en escolares cordobeses. Revista Española de Salud Pública, 72(3), 133-244.

Piaget, J. (1982). Psicología del niño. Madrid: Morata.

Ramírez-López, C. y Arcila-Rodríguez, W. (2013). Violencia, conflicto y agresividad en el escenario escolar. Educ. Educ., 16(3), 411-429.

Salinas, J. (2004). Cambios metodológicos con las TIC. Estrategias didácticas y entornos virutales de enseñanza aprendizaje. Revista Electrónica de Tecnología Educativa, 30, 1-20.

Sarmiento, M. (2007). La enseñanza de las matemáticas y las NTIC. Una estrategia de formacion permanente. [Tesis de doctorado, Universitat Rovira i Virgili]. Repositorio institucional URV. http://hdl.handle.net/10803/8927
Serra Grima, J. (2001). Corazón y ejercicio físico en la infancia y adolescencia. Barcelona: Masson. https:// www.tirant.com/libreria/libro/corazon-y-ejercicio-fisico-en-la-infancia-y-adolescencia-j-r-serragrima-9788445811283

Stake, R. (1999). Investigación con estudio de casos. Madrid: Morata.

Taylor, S. y Bogdan, R. (1986). Introducción a métodos cualitativos de investigación. Barcelona: Paidós.

Timón Benítez, L. y Hormigo Gamarro, F. (2010). Propuesta para la mejora de la resistencia en la educación física en la etapa secundaria. Sevilla: Wanceulen.

Treanor, L. (1998). Middle school student's perceptions of coeducational and same-sex Physical Education classes. Journal of Teaching Physical Education, 18, 43-56.

Vigotsky, L. (1931). Historia del desarrollo de las funciones psíquicas superiores. Vol. 3. La Habana: Comisión Editorial para la Edición en Lengua Rusa: Pueblo y Educación. https://edisciplinas.usp.br/pluginfile. php/289941/mod_folder/content/.../Tomo\%203.pdf?

Vuori, I. (1987). Ejercicio físico y salud. Foro Mundial de la Salud, 8(2), 135-159. https://apps.who.int/iris/ handle/10665/51807

Zimmerman, M., Campomar, G., Fulugonio, M. y Castro, H. (2013). Problemáticas actuales de la educación física: estudio exploratorio sobre la formación e inserción profesional de los egresados universitarios. Ponencia presentada en el x Congreso Argentino y V Latinoamericano de Educación Física y Ciencias, La Plata, Argentina. 